\title{
PERSPECTIVE OPEN Cryo-EM advances in RNA structure determination
}

\author{
Haiyun Ma $\mathbb{D}^{1}$, Xinyu Jia ${ }^{1}$, Kaiming Zhang $\mathbb{D}^{2}$ and Zhaoming Su $\mathbb{D}^{1 凶}$
}

Cryo-electron microscopy (cryo-EM) has emerged as an unprecedented tool to resolve protein structures at atomic resolution. Structural insights of biological samples not accessible by conventional X-ray crystallography and NMR can be explored with cryoEM because measurements are carried out under near-native crystal-free conditions, and large protein complexes with conformational and compositional heterogeneity are readily resolved. RNA has remained underexplored in cryo-EM, despite its essential role in various biological processes. This review highlights current challenges and recent progress in using cryo-EM singleparticle analysis to determine protein-free RNA structures, enabled by improvement in sample preparation and integration of multiple structural and biochemical methods.

Signal Transduction and Targeted Therapy (2022)7:58 ; https://doi.org/10.1038/s41392-022-00916-0

The recent resolution revolution triggered by the development of direct electron detectors and other technical advances has allowed cryo-EM to break the previous resolution barrier(s) and led to exponential growth of near-atomic cryo-EM structures. ${ }^{1,2}$ More recently, several studies have achieved atomic-resolution cryo-EM reconstruction using either the next-generation hardware or the most up-to-date commercially available setup. ${ }^{3-5}$ Aside from single-particle analysis (SPA) that has gained tremendous popularity, other cryo-EM approaches have also benefited from the revolution, such as cryo-electron tomography (cryo-ET), 6,7 micro-electron diffraction (MicroED) ${ }^{8}$, and cryo-scanning transmission electron microscopy (cryo-STEM). ${ }^{9}$ Because cryo-EM SPA is featured by minimal amount of specimen under near-native crystal-free condition, automated data collection with continually increasing throughput, ${ }^{10}$ and comprehensive data processing pipelines capable of resolving structural heterogeneity, ${ }^{11}$ it has become a widely adopted structural biology technique for structural biologists whose portfolio used to have only X-ray crystallography and NMR. Whereas the above features make SPA particularly useful to determine structures of larger proteins and protein complexes $(>200 \mathrm{kDa})$, additional technical advances, especially the development of Volta phase plate (VPP), ${ }^{12}$ have pushed the limit of protein molecular weight to as low as $52 \mathrm{kDa}$ for structure determination by SPA. ${ }^{13}$ Readers interested in technical developments and current challenges in SPA are directed to a number of comprehensive reviews for further details. ${ }^{11,14-16}$

RNA plays an essential role in various important biological processes by folding and sustaining a three-dimensional (3D) structure in order to perform functions such as catalysis and gene regulation. ${ }^{17-19}$ It is estimated that about $85 \%$ of the human genome is transcribed into RNA, ${ }^{20}$ with more than $80 \%$ of the genome estimated to be biologically and functionally relevant, ${ }^{21}$ however, only 1.5 percent of the genome encodes proteins. ${ }^{21,22}$ In the Protein Data Bank (wwPDB), there are currently 183980 depositions of protein complexes, which has facilitated the development of accurate protein structure prediction algorithms. ${ }^{23}$ In contrast to our apparently better understanding of protein structures and functions, our knowledge of RNA structures remains scarce. There are currently 1569 protein-free RNA and 9790 protein-nucleic acid complex structures deposited in the PDB that accounts for only about $6 \%$ of the entire PDB deposition (Fig. 1a). The lack of RNA structures is most likely due to the intrinsic heterogeneity of RNAs caused by flexible ribose and phosphate backbone, weak long-range tertiary interactions, alternative conformations, and dynamics among multiple functional states, which pose great challenges to X-ray crystallography to obtain RNA crystals with high-resolution diffraction information, and to NMR to solve structures of RNAs larger than 100 nucleotides $(100 \mathrm{nt}){ }^{24,25}$ Although cryo-EM SPA has extended our accessibility to more challenging biological structures and systems, proteinfree RNA structures accrue at a much slower rate compared to proteins and protein-nucleic acid complexes in the Electron Microscopy Data Bank (EMDB). To date, there are only three protein-free RNA cryo-EM structures determined at $4 \AA$ or better resolution (Table 1), which accounts for $0.02 \%$ of the entire 17349 depositions in EMDB (Fig. 1b).

The scarcity of protein-free RNA cryo-EM structures may be attributed to the following reasons: (1) Effective approaches and/or pipelines are missing for obtaining properly folded RNAs with stable tertiary structures; (2) the majority of isolated functional RNAs are simply too small for facile visualization and reconstruction in standard SPA pipelines; (3) intrinsic heterogeneity in most RNA molecules greatly limits the attainable resolution by SPA. This review summarizes recent efforts that overcome these challenges and enrich the proteinfree RNA cryo-EM structure repertoire, including new approaches to obtain stable RNA structures for cryo-EM SPA and integration of multiple techniques to facilitate structural analysis when resolution is moderate. Cryo-EM structures of RNAs in RNPs such as ribosome, spliceosome, telomerase, and CRISPR complexes will not be discussed, as they are reviewed elsewhere. ${ }^{26-29}$ All RNA structures reviewed herein are cryo-EM structures of protein-free RNAs.

\footnotetext{
${ }^{1}$ The State Key Laboratory of Biotherapy, Department of Geriatrics and National Clinical Research Center for Geriatrics, West China Hospital, Sichuan University, Chengdu, Sichuan

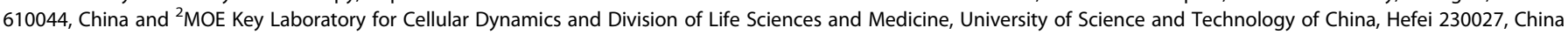
Correspondence: Zhaoming Su (zsu@scu.edu.cn)
}

Received: 22 November 2021 Revised: 27 January 2022 Accepted: 30 January 2022

Published online: 23 February 2022 
a

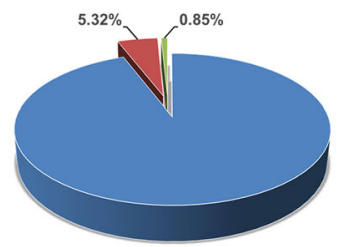

PDB depositions: 183,980 in total

— PDB depositions: 9,790 protein/nucleic acid complexes PDB depositions: 1,569 protein-free RNA

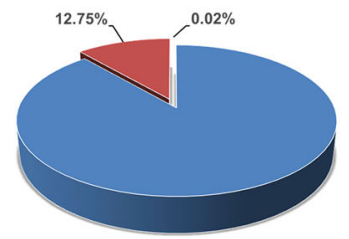

EMDB depositions: 17,349 in total

- EMDB depositions: 2,212 protein/nucleic acid complex

- EMDB depositions: 3 protein-free RNA at $4 \AA$ or better

Fig. 1 Statistics of protein-free RNA and protein-nucleic acid complex structures in PDB (a) and EMDB (b)

\begin{tabular}{|c|c|c|c|c|c|}
\hline ATP-TTR-3 (apo) ${ }^{37}$ & $130 / 42$ & 71,045 & 10 & Yes & 1501 \\
\hline F. nucleatum glycine riboswitch (with glycine) ${ }^{37}$ & $171 / 55$ & 35,578 & 7.4 & Yes & 415 \\
\hline F. nucleatum glycine riboswitch (apo) ${ }^{37}$ & $171 / 55$ & 20,269 & 10 & Yes & 840 \\
\hline hc16 ligation product ${ }^{37}$ & $349 / 112$ & 29,191 & 10 & Yes & 1055 \\
\hline Tetrahymena L-21 Scal ribozyme (apo $)^{37}$ & $388 / 125$ & 74,621 & 6.8 & Yes & 553 \\
\hline HIV-1 DIS ${ }^{45}$ & $94 / 30$ & 24,934 & 9 & No & N/A \\
\hline Mycobacterium SAM-IV riboswitch (with SAM) ${ }^{37}$ & $119 / 40$ & 225,303 & 4.8 & No & 303 \\
\hline Mycobacterium SAM-IV riboswitch (apo) ${ }^{37}$ & $119 / 40$ & 260,244 & 4.7 & No & 238 \\
\hline B. subtilis glyQS T-box (with tRNA ${ }^{\text {Gly }}{ }^{46}$ & $244 / 74$ & 189,361 & 4.9 & No & 232 \\
\hline L. lactis L1 LtrA-depleted intron RNA ${ }^{33}$ & $702 / 216$ & 102,522 & 4.5 & No & N/A \\
\hline $\mathrm{dENE}^{49}$ & $76 / 23$ & 69,623 & 8.7 & No & $\mathrm{N} / \mathrm{A}$ \\
\hline dENE-poly $\left(A_{28}\right)^{49}$ & $104 / 32$ & 283,486 & 5.6 & No & N/A \\
\hline Tetrahymena L-21 Scal ribozyme (apo $)^{42}$ & $388 / 118$ & 415,918 & 3.1 & No & 126 \\
\hline $\begin{array}{l}\text { Tetrahymena L-16 Scal ribozyme with two RNA oligonucleotide } \\
\text { substrates }\end{array}$ & $407 / 125$ & 230,386 & 3.1 & No & 90 \\
\hline SARS-CoV-2 FSE ${ }^{44}$ & $88 / 28$ & 109,137 & 5.9 & No & 726 \\
\hline BMV $\operatorname{TLS}^{35}$ & $169 / 55$ & 128,266 & 4.3 & No & N/A \\
\hline Tetrahymena group I intron ${ }^{36}$ & $388 / 118$ & 82,575 & 3.0 & No & N/A \\
\hline
\end{tabular}

\section{NEW APPROACHES TO OBTAIN STABLE RNA STRUCTURES FOR SPA}

RNA X-ray crystallography utilizes strategies that introduce proteins such as U1A and L7Ae to bind to specific RNA motifs to stabilize RNA crystal lattices. ${ }^{30,31}$ Group II introns are ribozymes with endogenous protein partners that stabilize the dynamic RNA structures and facilitate RNA catalysis. ${ }^{32,33}$ The analogous approach has been reported by Qu et al. in the cryo-EM structure of the Lactococcus lactis LtrB group II intron in complex with its intron-encoded protein LtrA at $3.8 \AA$ (Fig. 2a), in which they utilize a $60 \mathrm{kDa}$ RNA binding protein LtrA to stabilize the RNA structure and resolve $704 \mathrm{nt}(216 \mathrm{kDa})$ of the $902 \mathrm{nt}$ LtrB using cryo-EM SPA. ${ }^{34}$ Intriguingly, 3D classification yielded one minor class that resembled the $L$ trB intron alone with four times fewer particles than the LtrB-LtrA RNP, which generated a protein-free RNA structure at $4.5 \AA$. The disordered LtrA binding site in LtrAdepleted RNA structure validates the role of LtrA to assist folding and to enhance the stability of LtrB intron structure as previously described. $^{34,35}$

Bonilla and colleagues used cryo-EM SPA to solve the viral tRNA-like structure (TLS) of bromo mosaic virus (BMV) at $4.3 \AA$ resolution. ${ }^{36}$ Unexpectedly, the "tRNA-like" L-shape was not readily observed in protein-free BMV TLS. Comparison of the TLS alone with the complex structure of TLS and tyrosyl-tRNA synthetase (TyrRS) indicates that TLS undergoes drastic conformational changes to resemble "tRNA-like" shape in order to bind to TyrRS. This is a great example illustrating the importance of studying protein-free RNAs to provide insights of required conformational rearrangement upon protein binding.

Liu and coworkers recently reported a nanostructure assembly strategy called "RNA oligomerization-enabled cryo-EM via installing kissing-loops (ROCK)", to obtain homomeric self-assembled dimers and trimers of protein-free RNAs for SPA at near-atomic to subnanometer resolutions. ${ }^{37}$ Self-assembly is achieved by 
insertions of kissing-loop sequence in the functionally nonessential peripheral stem loops, which makes the assembled RNA two- to three-times larger than the monomeric RNA (Fig. 2b). While this strategy could be potentially useful for RNAs too small for particle picking and orientation alignment in cryo-EM SPA, it may also have an artificial impact on RNA dynamics after

a

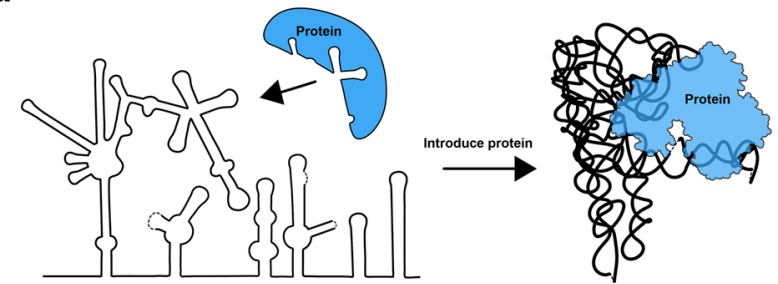

b

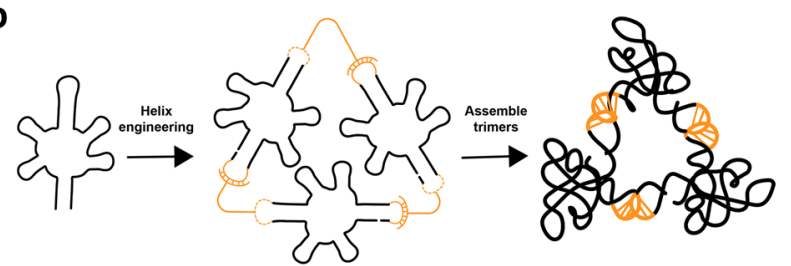

Fig. 2 New approaches to obtain stable RNA structures for cryo-EM SPA. (a) Protein binding partners can be introduced to stabilize RNA structures. (b) Self-assembled homomeric RNA nanostructures potentially allow cryo-EM SPA studies of relative small RNAs oligomerization. Prior knowledge of RNA structure-function relationship is also required to guide insertions of kissing-loop sequences.

\section{NEW WORKFLOW THAT ACCELERATES THE DETERMINATION OF RNA CRYO-EM STRUCTURES}

Kappel et al. recently developed an accelerated RNA structure determination workflow, named "Ribosolve" (Fig. 3a), that integrates native gel analysis, mutate-and-map by next generation sequencing (M2-seq), cryo-EM SPA, and auto-DRRAFTER RNA modeling. ${ }^{38}$ In this workflow, native gel analysis enables quick identification of optimal RNA refolding conditions. RNAs that form sharp bands in native gels are subjected to M2-seq experiments, ${ }^{39}$ in which secondary structures are determined to both assess the homogeneity of folded RNA structures and to assist RNA modeling. Cryo-EM SPA allowed the rapid determination of 11 previously unknown RNA structures at resolution ranging from 4.7 to $10 \AA$, which revealed explicit RNA features of major and minor grooves. Seven structures were determined with VPP for contrast enhancement. Finally, auto-DRRAFTER was developed based on the previous DRRAFTER ${ }^{40}$ to utilize secondary structures determined by M2-seq to build 3D RNA models guided by cryo-EM density, because de novo RNA modeling can be very challenging under such resolution. The estimated coordinate root mean square deviation (RMSD) accuracy was between 3.3 and $6.3 \AA$ as predicted by modeling convergence, which is sufficient for revealing RNA overall fold. This workflow enables accelerated RNA cryo-EM structure determinations by introducing methods to

\section{a}
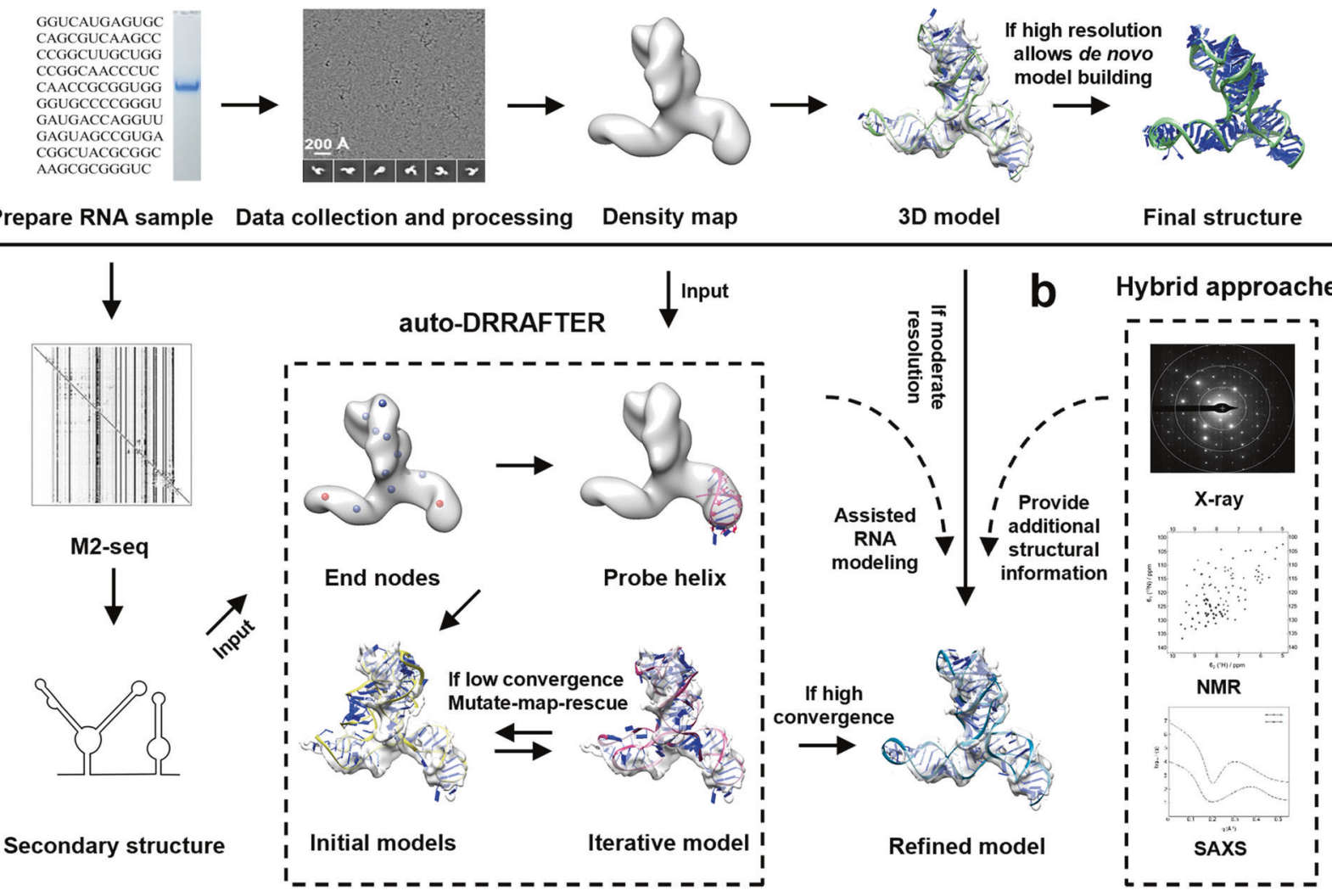

Fig. 3 Cryo-EM-guided RNA structure determination. a "Ribosolve" utilizes native gel analysis, secondary structure information, cryo-EM maps at moderate resolution, and RNA modeling algorithm to generate RNA models. $\mathbf{b}$ Cryo-EM maps at moderate resolution that do not allow de novo modeling can be combined with structural information determined by other techniques like X-ray crystallography, NMR and SAXS 
examine RNA folding and structural homogeneity, which can be used both to assist RNA modeling and to guide additional cryo-EM data collection for improved resolution.

A few applications of the "Ribosolve" workflow have been reported. SAM-IV riboswitch, a $40 \mathrm{kDa}$ RNA that recognizes S-adenosylmethionine (SAM), ${ }^{41}$ was initially resolved to $7 \AA$ resolution using cryo-EM SPA with VPP. Zhang et al. continued to collect additional cryo-EM data and achieved $3.7 \AA$ resolution for the apo state and $4.1 \AA$ for the SAM-bound state allowing unambiguous ligand recognition. ${ }^{42}$ The final data sets that generated cryo-EM structures of both states at near-atomic resolution were collected without VPP. This is likely because the stronger electron scattering of phosphate backbones has provided sufficient contrast for visualization of this small RNA. At $3.7 \AA$ resolution, only a portion of the base pairs is resolved, indicating that higher resolution is required to allow de novo modeling. Nonetheless, this study has demonstrated the capability of cryo-EM SPA to facilitate structure determination at near-atomic resolution for RNA that is beyond the current molecular weight limit of $52 \mathrm{kDa}$ for protein. ${ }^{13}$

Recently, Su and colleagues solved the cryo-EM structures of fulllength Tetrahymena ribozyme in both apo and substrate-bound states at $3.1 \AA$ resolution. ${ }^{43}$ Group I self-splicing intron of Tetrahymena thermophila was discovered as the first ribozyme and has since become an unprecedented model system to study RNA catalysis and structure-function relationship. However, the fulllength Tetrahymena ribozyme structure remains unknown for 40 years. After a $6.8 \AA$ cryo-EM map of the apo L-21 Tetrahymena ribozyme map was obtained by "Ribosolve", more data was collected to achieve a $3.1 \AA$ cryo-EM structure, in which the complete peripheral region was explicitly resolved and two previously unforeseen tertiary interactions that allosterically regulate catalysis were identified. The substrate-bound state revealed catalytic mechanism, conformational changes of the internal guide sequence, and local shifts of phosphate, nucleobase, and metal ions upon substrate binding. This study provided a complete structural view of the Tetrahymena ribozyme and a great example of dissecting the RNA structure-function relationship by cryo-EM.

During the SARS-CoV-2 pandemic, cryo-EM SPA has played essential roles in unveiling the structural basis of viral infection, replication, and other stages in the virus life cycle. ${ }^{44}$ Using "Ribosolve", Zhang et al. resolved the $6.9 \AA$ cryo-EM structure of the frameshifting stimulus elements (FSE), a highly conserved $88 \mathrm{nt}$ RNA that is essential for the balanced expression of important viral proteins in the SARS-CoV-2 genome. ${ }^{45}$ It is also a potential candidate target for antiviral drugs such as small molecules and antisense oligonucleotides (ASO). Under the guidance of cryo-EM map, the RNA model was built with an estimated RMSD accuracy of $5.9 \AA$ predicted by RNA modeling convergence following the "Ribosolve" pipeline. ${ }^{38}$ Subsequently, ASOs targeting FSE were designed and their antiviral activities were verified in vitro and at cellular level. This study features the smallest $28 \mathrm{kDa}$ protein-free RNA studied by cryo-EM and resolving the tertiary structure of this key RNA element of SARS-CoV-2 potentially speeds up the development of new therapies for COVID-19.

\section{HYBRID APPROACHES TO FACILITATE RNA STRUCTURAL ANALYSIS}

Zhang et al. used cryo-EM SPA to resolve a $30 \mathrm{kDa}$ HIV-1 dimer initiation site RNA (DIS) at $9 \AA$ resolution. The cryo-EM map helps restraining NMR model refinement to derive atomic ensembles of this RNA duplex to identify a flipped-out nucleotide at both ends of the duplex. ${ }^{46}$ Molecular dynamics (MD) simulations of multiple time points on simulated cryo-EM data of DIS suggest that the intrinsic RNA structural heterogeneity limits cryo-EM SPA from achieving higher resolution for the DIS RNA.

$\mathrm{Li}$ et al. resolved a $4.9 \AA$ cryo-EM structure of the full-length Tbox-tRNA ${ }^{\text {Gly }}$ complex. $^{47}$ Although the central tRNA ${ }^{\text {Gly }}$ is better resolved to $4.1 \AA$, featured by separated density of some phosphate and ribose groups, the outer T-box is only resolved to $6 \AA$. This moderate resolution poses great challenge for RNA modeling, especially in the newly identified $66 \mathrm{nt} 3^{\prime}$-discriminator region with previously unknown structural features. They tackled this problem by determining a co-crystal structure of tRNA ${ }^{\text {Gly }}$ in complex with the $3^{\prime}$-discriminator from another bacterial species at $2.7 \AA$ resolution. The final model of the full length T-box-tRNA ${ }^{\text {Gly }}$ complex was generated by combining the $3^{\prime}$-discriminator RNA model with the previously known crystal structure of tRNA Gly and $5^{\prime}$-Stem I complex and refined into the cryo-EM density. ${ }^{48,49}$ Cryo-EM 3D classification indicated that structural heterogeneity of the T-box 5'-Stem I and 3 '-discriminator regions may have limited the overall resolution.

In another study, Torabi et al. resolved cryo-EM maps of a double element for nuclear expression (dENE) before and after poly $(\mathrm{A})$ binding at subnanometer resolution. ${ }^{50}$ The cryo-EM structures show an overall similar architecture compared to a previous high-resolution crystal structure except for an $\sim 23^{\circ}$ curvature at the end of one ENE, indicating that crystal packing may not reveal minor structural dynamics in solution. Small angle $X$-ray scattering (SAXS) reveals a global shape that is consistent with both cryo-EM and crystal structures.

In conclusion, these examples illustrate the combinations of different high-resolution structural biology techniques, such as X-ray crystallography and NMR, to facilitate the analysis of cryo-EM RNA structures at moderate resolution (Fig. 3b). According to MD simulations and SPA 3D classification, the intrinsic structural heterogeneity is the major factor that limits resolution in cryo-EM reconstructions of RNA molecules.

\section{PERSPECTIVE}

Cryo-EM SPA can readily attain near-atomic resolution (between 2 and $4 \AA$ ) for proteins and protein complexes on a regular basis, which is sufficient to enable de novo model building. In contrast, RNA cryo-EM structures at near-atomic resolution are rare. The recent progress in RNA cryo-EM SPA has generated most RNA structures at a moderate resolution ranging from 4 to $10 \AA$, which are challenging for de novo modeling. Multiple strategies have been used synergistically to assist structural analysis of these RNA cryo-EM structures, including hybrid approaches combining RNA modeling, NMR, and X-ray crystallography. In order to facilitate structural analysis based on cryo-EM structures, it is critical to identify and overcome the major factors that limit cryo-EM SPA to attain RNA structures at near-atomic resolution.

In cryo-EM SPA, an overall temperature factor, or $B$-factor, derived from the correlation between the number of particles used for reconstruction and the achieved resolution, is used to evaluate the quality of the cryo-EM data (i.e. low $B$-factor indicates high data quality because fewer particles are required to achieve certain resolution). ${ }^{51}$ The $B$-factor values calculated for RNAs between 40 and $125 \mathrm{kDa}$ are all greater than $200 \AA^{2}$ (Table 1), whereas sub- $2 \AA$ cryo-EM structures of proteins normally have a $B$ factor around $60 \AA^{2,52}$. Interestingly, $B$-factor values seem much larger for RNA structures reconstructed from cryo-EM data with VPP than those without VPP, indicating that VPP does not seem to help reducing the $B$-factor for RNA cryo-EM structures, albeit it significantly improves image contrast.

Biological samples are susceptible to radiation damage and limited electron exposure is used in Cryo-EM that leads to low contrast and signal-to-noise ratio (SNR), which will deteriorate the accuracy of particle alignment in SPA. ${ }^{5,54}$ The optimal exposure of cryo-EM SPA for protein has been determined to be $\sim 20 \mathrm{e}^{-} / \AA^{2}$, although higher dose could be used when exposure filtering is applied to enhance SNR at lower spatial frequencies in order to minimize alignment errors of particle orientations. ${ }^{55}$ Fujiyoshi et al. have previously found that tRNA crystals embedded in glycerol are at least 4 times less susceptible to radiation damage compared 
to protein crystals in glycerol based on the critical dose curve when exposed to electrons at room temperature. ${ }^{56}$ The radiation damage effect on RNAs embedded in vitrified ice under cryogenic temperature has not been characterized in detail. As more RNA cryo-EM structures are determined with continuously improving resolution, radiation damage and optimal exposure on RNA cryoEM structures need to be assessed in order to guide optimal electron dose in cryo-EM data collection. For example, if RNA is less susceptible to radiation damage, higher total electron doses could be used in RNA cryo-EM SPA to enhance image contrast and SNR which will lead to attenuation of alignment errors. This may reduce the $B$-factor since small particles like RNAs are particularly sensitive to alignment errors. ${ }^{57}$

A number of other factors may have an impact on the $B$-factor, such as ice thickness, beam-induced motion (BIM), and intrinsic heterogeneity of the RNA molecules. To eliminate ice thickness variations, in which thicker ice will lead to decreased image contrast and SNR, novel sample preparation instruments have been developed and are currently optimized to reproducibly generate thin ice. For example, "Spotiton" can deliver as little as a few nanoliters of samples onto the grid while it plunges. ${ }^{58}$ The reduced time window between spotting and plunging also attenuates the air-water interface problem, ${ }^{59}$ which causes other issues in SPA such as preferred orientation and sample denaturation (reviewed elsewhere ${ }^{60}$ ).

BIM has been previously shown to be drastically reduced by the implementation of various supporting films and foils. ${ }^{61}$ In particular, the amorphous nickel-titanium alloy (ANTA) film has been developed to reduce BIM. ${ }^{62}$ More recently, BIM has been minimized utilizing ultrastable gold foils on gold grids with specific hole diameter that ameliorate stress in the vitrified ice, named HexAuFoil.63,64 The drastic translational and rotational motions discovered in the first few frames that carry the highest resolution information can also be ameliorated by finer intervals (i.e. more frames) to improve motion correction accuracy. The resulting lower contrast and SNR of each frame can be potentially compensated by VPP or weighted denoising algorithms that should theoretically enhance SNR at low spatial frequencies. ${ }^{65,66}$

RNA may naturally adopt higher $B$-factor than protein due to its intrinsic heterogeneity and dynamics. Structural study is primarily facilitated by the identification of RNAs with conformational homogeneity and stable 3D structures, as demonstrated by native gel analysis and M2-seq in the "Ribosolve" workflow. ${ }^{37}$ Additional information from computational analysis, such as phylogenetic analysis and predictions of long-range tertiary interactions (e.g. pseudoknot formations, minor-groove interactions, etc.) will further enable the identification of conformational homogeneous RNAs with stable 3D structures. ${ }^{17,67}$ It is noteworthy that cryo-EM provides the unique opportunity of resolving conformational heterogeneity. Recent advance in automated data collection strategies that uses beam-shift instead of mechanical stage movement for each exposure, ${ }^{68}$ combined with image processing algorithm that compensates the aberrations caused by beam-shift, ${ }^{69}$ enable significantly increased throughput of data collection. As more particles are collected within the same time frame, classification of multiple conformations become realistic using existing algorithms such as Relion multibody refinement, ${ }^{70}$ cryoSPARC 3D variability analy$\mathrm{sis}^{71}$ and the emerging neural network-based 3D heterogeneity analysis, ${ }^{72}$ to eventually resolve RNA structures and their intrinsic heterogeneity and dynamics.

We are in an exciting era for structural biology, in which the emerging deep learning structure prediction algorithms have enabled reliable structure predictions of proteins and protein complexes in eukaryotes including human..$^{23,73-76}$ However, these algorithms rely heavily on the plentifulness of experimental data. Although a recent study has reported a scoring system trained by very few existing RNA structures using machine learning algorithm to improve RNA structure prediction accuracy, ${ }^{77}$ the lack of experimental RNA structures remains to be the major barrier for precise RNA structure prediction. Recent progress in the field of RNA cryo-EM demonstrates how cryo-EM SPA complements X-ray crystallography and NMR to provide insightful structural information. Continued advances in technical and experimental developments of cryo-EM will allow accelerated structural findings previously not accessible by X-ray crystallography and NMR, and generate RNA structures at near-atomic resolution on a more regular basis. This will provide structural insights to deepen our understanding of RNA structure-function relationships as well as facilitating precise RNA structure predictions in the near future.

\section{ACKNOWLEDGEMENTS}

The authors are grateful to Janusz Bujnicki and Sebastian Glatt for their critical reading and insightful comments of the manuscript. This work was supported by Natural Science Foundation of China (NSFC) 32070049 and 82041016 (ZS), Start-up funding by the University of Science and Technology of China KY9100000032, and KJ2070000080 (KZ), and Sichuan University start-up funding 20822041D4057 (ZS).

\section{AUTHOR CONTRIBUTIONS}

K.Z. and Z.S. calculated $B$-factor values, all participated in writing the manuscript. All authors have read and approved the article.

\section{ADDITIONAL INFORMATION}

Competing interests: The authors declare no competing interests.

\section{REFERENCES}

1. Faruqi, A. R. \& Henderson, R. Electronic detectors for electron microscopy. Curr. Opin. Struct. Biol. 17, 549-555 (2007).

2. Kuhlbrandt, W. Biochemistry. The resolution revolution. Science 343, 1443-1444 (2014).

3. Nakane, T. et al. Single-particle cryo-EM at atomic resolution. Nature 587, 152-156 (2020)

4. Yip, K. M., Fischer, N., Paknia, E., Chari, A. \& Stark, H. Atomic-resolution protein structure determination by cryo-EM. Nature 587, 157-161 (2020).

5. Zhang, K., Pintilie, G. D., Li, S., Schmid, M. F. \& Chiu, W. Resolving individual atoms of protein complex by cryo-electron microscopy. Cell Res. 30, 1136-1139 (2020).

6. Doerr, A. Cryo-electron tomography. Nat. Methods 14, 34-34 (2017).

7. Koning, R. I., Koster, A. J. \& Sharp, T. H. Advances in cryo-electron tomography for biology and medicine. Ann. Anat. 217, 82-96 (2018).

8. Nannenga, B. L. \& Gonen, T. The cryo-EM method microcrystal electron diffraction (MicroED). Nat. Methods 16, 369-379 (2019).

9. Wolf, S. G. \& Elbaum, M. CryoSTEM tomography in biology. Methods Cell Biol. 152, 197-215 (2019).

10. Tan, Y. Z., Cheng, A., Potter, C. S. \& Carragher, B. Automated data collection in single particle electron microscopy. Microscopy 65, 43-56 (2015).

11. Danev, R., Yanagisawa, H. \& Kikkawa, M. Cryo-electron microscopy methodology: Current aspects and future directions. Trends Biochem. Sci. 44, 837-848 (2019).

12. Danev, R., Buijsse, B., Khoshouei, M., Plitzko, J. M. \& Baumeister, W. Volta potential phase plate for in-focus phase contrast transmission electron microscopy. Proc. Natl Acad. Sci. USA 111, 15635-15640 (2014).

13. Fan, $X$. et al. Single particle cryo-EM reconstruction of $52 \mathrm{kDa}$ streptavidin at 3.2 Angstrom resolution. Nat. Commun. 10, 2386 (2019).

14. Lyumkis, D. Challenges and opportunities in cryo-EM single-particle analysis. J. Biol. Chem. 294, 5181-5197 (2019).

15. Vinothkumar, K. R. \& Henderson, R. Single particle electron cryomicroscopy: Trends, issues, and future perspective. Q. Rev. Biophys. 49, e13 (2016).

16. Glaeser, R. M. How good can single-particle cryo-EM become? What remains before it approaches its physical limits? Annu. Rev. Biophys. 48, 45-61 (2019).

17. Miao, Z. \& Westhof, E. RNA structure: Advances and assessment of 3D structure prediction. Annu. Rev. Biophys. 46, 483-503 (2017).

18. Cech, T. R. The RNA worlds in context. Cold Spring Harb. Perspect. Biol. 4, a006742 (2012).

19. Cech, T. R. \& Steitz, J. A. The noncoding RNA revolution-trashing old rules to forge new ones. Cell 157, 77-94 (2014).

20. Djebali, S. et al. Landscape of transcription in human cells. Nature 489, 101-108 (2012).

21. Lander, E. S. et al. Initial sequencing and analysis of the human genome. Nature 409, 860-921 (2001). 
22. Birney, E. et al. Identification and analysis of functional elements in $1 \%$ of the human genome by the ENCODE pilot project. Nature 447, 799-816 (2007).

23. Baek, M. et al. Accurate prediction of protein structures and interactions using a three-track neural network. Science 373, 871-876 (2021).

24. Zhang, H. \& Keane, S. C. Advances that facilitate the study of large RNA structure and dynamics by nuclear magnetic resonance spectroscopy. Wiley Interdiscip. Rev.: RNA 10, e1541 (2019).

25. Zhang, J. \& Ferre-D'Amare, A. R. New molecular engineering approaches for crystallographic studies of large RNAs. Curr. Opin. Struct. Biol. 26, 9-15 (2014).

26. Feigon, J., Chan, H. \& Jiang, J. Integrative structural biology of Tetrahymena telomerase-insights into catalytic mechanism and interaction at telomeres. FEBS J. 283, 2044-2050 (2016).

27. Fica, S. M. \& Nagai, K. Cryo-electron microscopy snapshots of the spliceosome: structural insights into a dynamic ribonucleoprotein machine. Nat. Struct. Mol. Biol. 24, 791-799 (2017).

28. Jiang, F. \& Doudna, J. A. The structural biology of CRISPR-Cas systems. Curr. Opin. Struct. Biol. 30, 100-111 (2015).

29. von Loeffelholz, O. et al. Focused classification and refinement in high-resolution cryo-EM structural analysis of ribosome complexes. Curr. Opin. Struct. Biol. 46, 140-148 (2017).

30. Ferre-D'Amare, A. R., Zhou, K. \& Doudna, J. A. A general module for RNA crystallization. J. Mol. Biol. 279, 621-631 (1998).

31. Huang, L. \& Lilley, D. M. The molecular recognition of kink-turn structure by the L7Ae class of proteins. RNA 19, 1703-1710 (2013).

32. Lambowitz, A. M. \& Zimmerly, S. Group II introns: Mobile ribozymes that invade DNA. Cold Spring Harb. Perspect. Biol. 3, a003616 (2011).

33. Haack, D. B. et al. Cryo-EM structures of a group II intron reverse splicing into DNA. Cell 178, 612-623 e612 (2019).

34. Qu, G. et al. Structure of a group II intron in complex with its reverse transcriptase. Nat. Struct. Mol. Biol. 23, 549 (2016).

35. Noah, J. W. \& Lambowitz, A. M. Effects of maturase binding and Mg2+ concentration on group II intron RNA folding investigated by UV cross-linking. Biochemistry 42, 12466-12480 (2003).

36. Bonilla, S. L., Sherlock, M. E., MacFadden, A. \& Kieft, J. S. A viral RNA hijacks host machinery using dynamic conformational changes of a tRNA-like structure. Science 374, 955-960 (2021).

37. Liu, D., Thélot, F. A., Piccirilli, J. A., Liao, M. \& Yin, P. Sub-3 Å cryo-EM structure of RNA enabled by engineered homomeric self-assembly. Preprint at bioRxiv https:// doi.org/10.1101/2021.08.11.455951 (2021).

38. Kappel, K. et al. Accelerated cryo-EM-guided determination of three-dimensional RNA-only structures. Nat. Methods 17, 699-707 (2020).

39. Cheng, C. Y., Kladwang, W., Yesselman, J. D. \& Das, R. RNA structure inference through chemical mapping after accidental or intentional mutations. Proc. Natl Acad. Sci. USA 114, 9876-9881 (2017).

40. Kappel, K. et al. De novo computational RNA modeling into cryo-EM maps of large ribonucleoprotein complexes. Nat. Methods 15, 947-954 (2018).

41. Weinberg, $Z$. et al. Identification of 22 candidate structured RNAs in bacteria using the CMfinder comparative genomics pipeline. Nucleic Acids Res. 35, 4809-4819 (2007).

42. Zhang, K. et al. Cryo-EM structure of a $40 \mathrm{kDa}$ SAM-IV riboswitch RNA at $3.7 \mathrm{~A}$ resolution. Nat. Commun. 10, 5511 (2019).

43. Su, Z. et al. Cryo-EM structures of full-length Tetrahymena ribozyme at $3.1 \mathrm{~A}$ resolution. Nature 596, 603-607 (2021).

44. Leigh, K. E. \& Modis, Y. Imaging and visualizing SARS-CoV-2 in a new era for structural biology. Interface Focus. 11, 20210019 (2021).

45. Zhang, K. et al. Cryo-electron Microscopy and exploratory antisense targeting of the 28 ?kDa frameshift stimulation element from the SARS-CoV-2 RNA genome. Nat. Struct. Mol. Biol 28, 747-754 (2021).

46. Zhang, K. et al. Structure of the $30 \mathrm{kDa}$ HIV-1 RNA dimerization signal by a hybrid cryo-EM, NMR, and molecular dynamics approach. Structure 26, 490-498 e493 (2018).

47. Li, S. et al. Structural basis of amino acid surveillance by higher-order tRNA-mRNA interactions. Nat. Struct. Mol. Biol. 26, 1094-1105 (2019).

48. Grigg, J. C. \& Ke, A. Structural determinants for geometry and information decoding of tRNA by T box leader RNA. Structure 21, 2025-2032 (2013).

49. Zhang, J. \& Ferre-D'Amare, A. R. Co-crystal structure of a T-box riboswitch stem I domain in complex with its cognate tRNA. Nature 500, 363-366 (2013).

50. Torabi, S. F. et al. Structural analyses of an RNA stability element interacting with poly(A). Proc. Natl. Acad. Sci. USA 118, e2026656118 (2021).

51. Rosenthal, P. B. \& Henderson, R. Optimal determination of particle orientation, absolute hand, and contrast loss in single-particle electron cryomicroscopy. J. Mol. Biol. 333, 721-745 (2003).

52. Tan, Y. Z. et al. Sub-2 A Ewald curvature corrected structure of an AAV2 capsid variant. Nat. Commun. 9, 3628 (2018)
53. Glaeser, R. M. Limitations to significant information in biological electron microscopy as a result of radiation damage. J. Ultrastruct. Res. 36, 466-482 (1971).

54. Henderson, R. The potential and limitations of neutrons, electrons, and X-rays for atomic resolution microscopy of unstained biological molecules. Q. Rev. Biophys. 28, 171-193 (1995).

55. Grant, T. \& Grigorieff, N. Measuring the optimal exposure for single particle cryoEM using a 2.6 A reconstruction of rotavirus VP6. elife 4, e06980 (2015).

56. Fujiyoshi, Y., Uyeda, N., Morikawa, K. \& Yamagishi, H. Electron microscopy of tRNA crystals. II. $4 \mathrm{~A}$ resolution diffraction pattern and substantial stability to radiation damage. J. Mol. Biol. 172, 347-354 (1984).

57. Henderson, R. et al. Tilt-pair analysis of images from a range of different specimens in single-particle electron cryomicroscopy. J. Mol. Biol. 413, 1028-1046 (2011).

58. Jain, T., Sheehan, P., Crum, J., Carragher, B. \& Potter, C. S. Spotiton: A prototype for an integrated inkjet dispense and vitrification system for cryo-TEM. J. Struct. Biol. 179, 68-75 (2012)

59. Noble, A. J. et al. Reducing effects of particle adsorption to the air-water interface in cryo-EM. Nat. Methods 15, 793-795 (2018).

60. Glaeser, R. M. Proteins, interfaces, and cryo-Em grids. Curr. Opin. Colloid Interface Sci. 34, 1-8 (2018).

61. Russo, C. J. \& Passmore, L. A. Progress towards an optimal specimen support for electron cryomicroscopy. Curr. Opin. Struct. Biol. 37, 81-89 (2016).

62. Huang, X. et al. Reprint of "Amorphous nickel titanium alloy film: A new choice for cryo electron microscopy sample preparation". Prog. Biophys. Mol. Biol. 160, 5-15 (2021).

63. Russo, C. J. \& Passmore, L. A. Electron microscopy: Ultrastable gold substrates for electron cryomicroscopy. Science 346, 1377-1380 (2014).

64. Naydenova, K., Jia, P. \& Russo, C. J. Cryo-EM with sub-1 A specimen movement. Science 370, 223-226 (2020).

65. Bhamre, T., Zhang, T. \& Singer, A. Denoising and covariance estimation of single particle cryo-EM images. J. Struct. Biol. 195, 72-81 (2016).

66. Bepler, T., Kelley, K., Noble, A. J. \& Berger, B. Topaz-Denoise: General deep denoising models for cryoEM and cryoET. Nat. Commun. 11, 5208 (2020).

67. Ponce-Salvatierra, A. et al. Computational modeling of RNA 3D structure based on experimental data. Biosci. Rep. 39, BSR20180430 (2019).

68. Cheng, A. et al. High resolution single particle cryo-electron microscopy using beam-image shift. J. Struct. Biol. 204, 270-275 (2018).

69. Zivanov, J. et al. New tools for automated high-resolution cryo-EM structure determination in RELION-3. Elife 7, e42166 (2018).

70. Scheres, S. H. Processing of structurally heterogeneous cryo-EM data in RELION. Methods Enzymol. 579, 125-157 (2016).

71. Punjani, A. \& Fleet, D. J. 3D variability analysis: Resolving continuous flexibility and discrete heterogeneity from single particle cryo-EM. J. Struct. Biol. 213, 107702 (2021)

72. Zhong, E. D., Bepler, T., Berger, B. \& Davis, J. H. CryoDRGN: Reconstruction of heterogeneous cryo-EM structures using neural networks. Nat. Methods 18, 176-185 (2021).

73. Humphreys, I. R. et al. Computed structures of core eukaryotic protein complexes. Science 374, eabm4805 (2021).

74. Jumper, J. et al. Highly accurate protein structure prediction with AlphaFold. Nature 596, 583-589 (2021).

75. Tunyasuvunakool, K. et al. Highly accurate protein structure prediction for the human proteome. Nature 596, 590-596 (2021).

76. Evans, R. et al. Protein complex prediction with AlphaFold-Multimer. Preprint at bioRxiv https://doi.org/10.1101/2021.10.04.463034 (2021).

77. Townshend, R. J. L. et al. Geometric deep learning of RNA structure. Science $\mathbf{3 7 3}$ 1047-1051 (2021)

Open Access This article is licensed under a Creative Commons Attribution 4.0 International License, which permits use, sharing, adaptation, distribution and reproduction in any medium or format, as long as you give appropriate credit to the original author(s) and the source, provide a link to the Creative Commons license, and indicate if changes were made. The images or other third party material in this article are included in the article's Creative Commons license, unless indicated otherwise in a credit line to the material. If material is not included in the article's Creative Commons license and your intended use is not permitted by statutory regulation or exceeds the permitted use, you will need to obtain permission directly from the copyright holder. To view a copy of this license, visit http://creativecommons. org/licenses/by/4.0/.

(c) The Author(s) 2022 